\title{
Light Dark Matter at Neutrino Experiments
}

\author{
Yohei Ema, ${ }^{1,2}$ Filippo Sala, ${ }^{1}$ and Ryosuke Sato ${ }^{1}$ \\ ${ }^{1}$ DESY, Notkestraße 85, D-22607 Hamburg, Germany \\ ${ }^{2}$ KEK Theory Center, Tsukuba 305-0801, Japan
}

(Received 26 November 2018; revised manuscript received 13 March 2019; published 8 May 2019)

\begin{abstract}
Sub-GeV dark matter particles up-scattered by cosmic rays gain enough kinetic energy to pass the thresholds of large volume detectors on Earth. We then use public Super-Kamiokande and MiniBooNE data to derive a novel limit on the scattering cross section of dark matter with electrons that extends down to sub-keV masses, closing a previously allowed wide region of parameter space. We finally discuss search strategies and prospects at existing and planned neutrino facilities.
\end{abstract}

DOI: 10.1103/PhysRevLett.122.181802

Introduction.-Evidence for dark matter (DM) is all based on its gravitational effects; other possible interactions of this unexplained component of the Universe are currently unknown. Some information about these interactions is obtained by direct detection (DD) experiments, which aim at observing the scattering of DM particles off standard model (SM) targets [1]. This has resulted in a huge experimental effort that, in the absence of any clear DM detection, has set strong limits on the DM-SM interactions for DM masses above a few $\mathrm{GeV}$; see, e.g., Refs. [2-4].

This situation is accompanied by the severe bounds that the LHC is putting on $\mathrm{TeV}$-scale new physics, which casts some doubt on natural solutions to the hierarchy problem; see, e.g., Ref. [5]. This undermines part of the motivation (i.e., the connection between naturalness and thermal relic DM) that leads to expect DM particles in the mass range where the above DD experiments are most sensitive. It is therefore no surprise that, especially in recent years, the community has vigorously pursued the exploration of lighter DM candidates, in terms of both model building and phenomenological tests (see Ref. [6] for a recent report).

The quest to determine the interactions of sub-GeV DM candidates is challenged by the low energy thresholds required by DD experiments. Indeed, the average DM velocity $v \approx 10^{-3}$ in the Milky Way halo implies that sub-GeV DM induces nuclear recoils below $O(\mathrm{keV})$, a value for which "standard" experiments like Xenon1T lose sensitivity. Analogously, the use of electron recoils in the same setups cannot probe DM masses below 1-10 MeV.

Published by the American Physical Society under the terms of the Creative Commons Attribution 4.0 International license. Further distribution of this work must maintain attribution to the author(s) and the published article's title, journal citation, and DOI. Funded by SCOAP .
A possibility to overcome this issue consists in devising new target materials and detector concepts that can be sensitive to very low energy recoils. This direction has been widely explored in recent years, resulting in the proposal and realization of several experiments (see again Ref. [6] for a review).

Another strategy to directly detect sub-GeV DM consists in relying on subdominant DM populations with much larger velocities so that their scattering off detectors can induce energetic recoils. A concrete example consists of ordinary DM particles up-scattered in high-temperature areas of the Sun $[7,8]$. The internal dynamics of nonminimal dark sectors can also result in relativistic dark species that could give signals in large detectors on Earth [9].

In this Letter we propose a new detection strategy of sub$\mathrm{GeV}$ dark matter based on the subdominant component with larger kinetic energy that is unavoidably generated by cosmic rays (CRs) that scatter off DM. Such up-scattered light DM can induce visible recoils in large volume detectors, by means of the very same interactions that accelerated it. Focusing on DM contact scatterings with electrons with cross section $\sigma_{e}$, we use public data of Super-Kamiokande (Super-K) and MiniBooNE to derive a new limit $\sigma_{e} \lesssim 10^{-(33-34)} \mathrm{cm}^{2}$. This limit constitutes the strongest existing constraint on DM lighter than a few $\mathrm{MeV}$, and it extends to DM masses much smaller than a keV. The possibility to probe CR interactions with light DM was first pointed out in a recent work [10] that derived constraints on DM from modifications of CR spectra. Our proposal tests directly the accelerated DM component by looking at its effects in detectors on Earth, rather than in CRs.

We finally discuss how searches for such a DM component could be optimized at Super-K, and the gain that one would achieve at large volume detectors with lower electron thresholds, like DUNE. Our proposal is robust against effects that typically hamper other detection 
strategies of light DM, like the possible existence of other SM-DM interactions or of small mass gaps in the dark sector.

From cosmic rays to DM scatterings on Earth.-A diffuse flux $\phi_{i}$ of particles with a scattering cross section $\sigma_{i}$ with DM, of mass $M_{\mathrm{DM}}$, induces a DM flux per solid angle

$$
\frac{d \phi_{\mathrm{DM}}}{d \Omega}\left(K_{\mathrm{DM}}, b, l\right)=\frac{J(b, l)}{M_{\mathrm{DM}}} \int d K_{i} \frac{d \phi_{i}}{d \Omega}\left(K_{i}\right) D_{i}^{\mathrm{DM}}\left(K_{i}, K_{\mathrm{DM}}\right) \sigma_{i},
$$

where $J(b, l)=\int_{\text {los }} d \ell \rho_{\mathrm{DM}}$ is the integral of the DM energy density $\rho_{\mathrm{DM}}$ over the line of sight in the direction of galactic coordinates $(b, l)$, and where we assume for simplicity that the CR flux $\phi_{i}$ is homogeneous inside the region of integration, which we take as customary as a cylinder centered on the Galactic Center (GC), with radius $R=10 \mathrm{kpc}$ and height $2 h=2 \mathrm{kpc} . D_{i}^{f}$ is a transfer function that encodes the energy spectrum of the particle $f$ induced by a scattering with particle $i$. Assuming $f$ to be initially at rest in the lab frame, its final kinetic energy reads

$$
\begin{aligned}
K_{f} & =K_{f}^{\max } \frac{1-\cos \theta}{2}, \\
K_{f}^{\max } & =\frac{2 m_{f}\left(K_{i}^{2}+2 m_{i} K_{i}\right)}{\left(m_{i}+m_{f}\right)^{2}+2 m_{f} K_{i}},
\end{aligned}
$$

where $\theta$ is the scattering angle in the center-of-mass (c.m.) frame. If the scattering is isotropic in the c.m. frame, then

$$
D_{i}^{f}=\frac{1}{K_{f}^{\max }\left(K_{i}\right)} \Theta\left(K_{f}^{\max }\left(K_{i}\right)-K_{f}\right),
$$

where $\Theta$ denotes the Heaviside step function. The number of DM scatterings with the target particles $T$ in a volume (e.g., of a detector), per time per solid angle per final energy $K_{T}$ of the target particle, is then given by

$\frac{d N_{\mathrm{DM}}}{d t d \Omega d K_{T}}=\int d V d K_{\mathrm{DM}} n_{T} \sigma_{T} D_{\mathrm{DM}}^{T}\left(K_{\mathrm{DM}}, K_{T}\right) \frac{d \phi_{\mathrm{DM}}}{d \Omega}$,

where $\sigma_{T}$ is the scattering cross section of DM with the target particle and $n_{T}$ their number density.

As anticipated in the Introduction, we focus on cosmicray electrons. We use their flux as provided in Ref. [11] for energies between $2 \mathrm{MeV}$ and $90 \mathrm{GeV}$. To compute $J(b, l)$, we use a Navarro-Frenk-White DM density profile [12] with $\rho_{\mathrm{DM}}(r=8.5 \mathrm{kpc})=0.42 \mathrm{GeV} / \mathrm{cm}^{3}$ and $r_{s}=20 \mathrm{kpc}$. The precise choice of the profile has a mild impact on our treatment because we integrate over wide areas and because the DM flux is linear in $\rho_{\mathrm{DM}}$ (analogous in a broad sense to the case of DM decay). To give a benchmark, for $\sigma_{e}=10^{-30} \mathrm{~cm}^{2}$ we find that $\phi_{\mathrm{DM}}\left(K_{\mathrm{DM}}=1 \mathrm{GeV}\right)=$ $4.0 \times 10^{-6}\left(1.5 \times 10^{-3}\right) \mathrm{GeV}^{-1} \mathrm{sec}^{-1} \mathrm{~cm}^{-2}$ for $M_{\mathrm{DM}}=$ $0.1 \mathrm{MeV}(\mathrm{keV})$. See the Supplemental Material [13] for

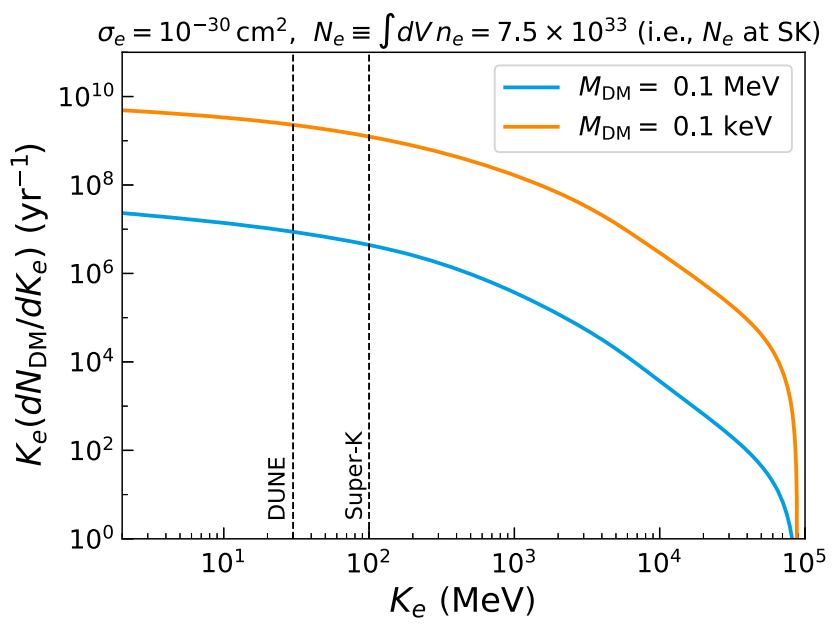

FIG. 1. Kinetic energy spectrum of electrons scattered by DM. The vertical dashed lines indicate the low energy thresholds considered at Super-K (100 MeV) and DUNE (30 MeV).

more details about the DM flux. Assuming a target material containing electrons $(T=e)$ and a DM-electron crosssection constant in energy results in the energy spectrum of the target electrons shown in Fig. 1. Note that, once produced, DM flux propagates without any further scatterings as the Galaxy is effectively transparent for the values of $\sigma_{e}$ of our interest.

An experiment that appears now in a privileged position to be sensitive to these events is Super-K, because of its unmatched large volume and because a sizable fraction of events survive the energy threshold $K_{e}>100 \mathrm{MeV}$ used in current analyses (see, e.g., Ref. [17]). As evident from Fig. 1, lower $K_{e}$ thresholds would allow us to collect more signal, but we are not aware of any existing experiment where the gain from the smaller thresholds is enough to compensate for the much smaller size. Thinking ahead, DUNE [18] will be ideally placed to test light DM via its unavoidable relativistic component, given its expected thresholds of $K_{e}>30 \mathrm{MeV}$ (see, e.g., Ref. [19]).

New constraints on light DM.-Super-K has recently performed a search for boosted DM in its "electron elastic scatter-like" events with $K_{e}>100 \mathrm{MeV}$ [17], in data corresponding to $161.9 \mathrm{kton}$ yr exposure. The results of Ref. [17] are directly applicable to our case, as we now explain. We use the total measured number of events reported in that Letter in the first energy bin $0.1<K_{e} / \mathrm{GeV}<1.33, N_{\mathrm{SK}}=4042$ to place a conservative limit on light DM as

$$
\epsilon \times N_{\mathrm{DM}}<N_{\mathrm{SK}},
$$

where $\epsilon=0.93$ is the signal efficiency as determined in Ref. [17]. We obtain $N_{\text {DM }}$ by integrating Eq. (4) over the total solid angle, 2628.1 days of data taking [17], and $K_{e}>100 \mathrm{MeV}$. We include Earth attenuation in the computation of $N_{\text {DM }}$ by writing the average kinetic energy loss of a DM particle as 


$$
\frac{d K_{\mathrm{DM}}}{d z}=-n_{e} \sigma_{e} \int d K K D_{\mathrm{DM}}^{e}\left(K_{\mathrm{DM}}, K\right)
$$

where $z$ is the depth from Earth's surface. We then assume for simplicity a constant $n_{e} \simeq 8 \times 10^{23} \mathrm{~cm}^{-3}$ (the averaged value over Earth) and integrate Eq. (6) from $z=0$ to $z_{\mathrm{SK}}$ that is the distance between Super-K and Earth's surface that depends on the direction of observation $\left(z_{\mathrm{SK}} \simeq 1 \mathrm{~km}\right.$ at the zenith), ignoring DM deflections. We use the DM kinetic energy obtained this way in Eq. (4) to determine the events in the detector. [An analogous treatment has been shown to be a good and conservative approximation of the numerical results in Ref. [20] ("method b").]

The resulting limit on an energy-independent $\sigma_{e}$ is shown as a shaded area in Fig. 2. The even more conservative limit obtained by working with $h=100 \mathrm{pc}$, instead of $1 \mathrm{kpc}$, is also shown as a thin line for comparison. The limits coming from the two higher energy bins given in Ref. [17] result in weaker constraints than the one we show. Our procedure sets limits in the ballpark of $\sigma_{e}<10^{-33} \mathrm{~cm}^{2}$ for $M_{\mathrm{DM}} \lesssim 0.1 \mathrm{keV}$ that slowly degrade at larger masses.

The behaviors of our exclusions can be analytically understood as follows. For $10 \mathrm{MeV} \gtrsim M_{\mathrm{DM}} \gtrsim 0.1 \mathrm{keV}$ all cosmic rays with energy $>100 \mathrm{MeV}$ make the Super-K electrons pass the threshold so that the number of signal events $N_{\text {DM }}$ scales as $N_{\mathrm{DM}} \propto 1 / M_{\mathrm{DM}}$, following the DM number density. Then, since $N_{\mathrm{DM}} \propto \sigma_{e}^{2}$, the limit scales $\propto M_{\mathrm{DM}}^{1 / 2}$. For $M_{\mathrm{DM}} \lesssim 0.1 \mathrm{keV}$ the energy transferred from the CR electrons to the DM scales as $M_{\mathrm{DM}} K^{2} / m_{e}^{2}$. Therefore the minimal CR energy $K_{\text {min }}$ required to transfer at least $\approx 100 \mathrm{MeV}$ to the DM increases at lower masses as $M_{\mathrm{DM}}^{-1 / 2}$. Since the CR flux scales roughly as $\phi_{i} \propto K^{-3}$, its integral is proportional to $K_{\min }^{-2} \propto M_{\mathrm{DM}}$. This compensates for the $1 / M_{\mathrm{DM}}$ from the DM number density, resulting in roughly flat limits on $\sigma_{e}$. For $M_{\mathrm{DM}} \gtrsim 10 \mathrm{MeV}$, the energy transferred to the electrons in Super-K scales as $m_{e} K_{\mathrm{DM}}^{2} / M_{\mathrm{DM}}^{2}$; therefore the limit of integration in the $\mathrm{CR}$ energy is linear in $M_{\mathrm{DM}}$. Proceeding as before, we get $N_{\mathrm{DM}} \propto \sigma_{e}^{2} M_{\mathrm{DM}}^{-3}$, which leads to the scaling $\propto M_{\mathrm{DM}}^{3 / 2}$. As explained above, in the smallest and largest $M_{\mathrm{DM}}$ regions shown in Fig. 2, the shape of our limits is driven by the CR electron of larger energies. Following Ref. [11], we have included their spectra only up to $90 \mathrm{GeV}$. For more than a decade above those energies the spectral index of electrons does not become softer [25], and this would, e.g., allow us to linearly extend our constraints to $M_{\mathrm{DM}}$ smaller and larger than what is shown in Fig. 2.

The region $\sigma_{e} \gtrsim 10^{-29} \mathrm{~cm}^{2}$ that is not excluded by Super-K is accessible at surface neutrino detectors. (See Ref. [26] for a recent list of such experiments with references, and Ref. [27] for a study of boosted DM at proto-DUNE.) To demonstrate this point, we use the MiniBooNE measurement [28] of two events of $\nu-e$ scattering, in a region defined by $\cos \theta_{e}>0.9$ along the

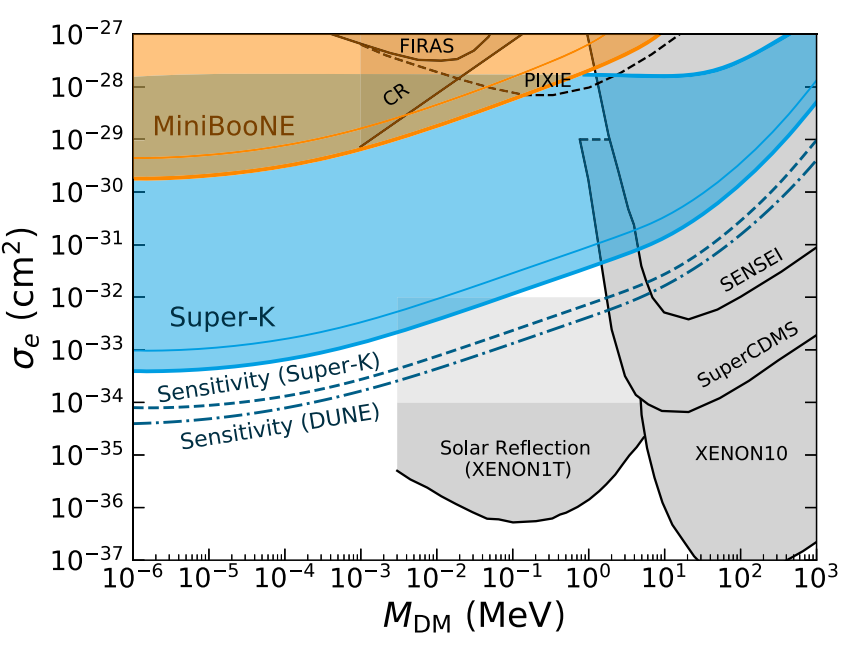

FIG. 2. Limits from Super-K (shaded blue) and MiniBooNE (shaded orange) and sensitivities at Super-K (blue dashed line) and DUNE (blue dotted-dashed line) on the DM-electron scattering cross section derived in this Letter. They correspond to a height of the cosmic-ray electron cylinder $h=1 \mathrm{kpc}$; the limits for a more conservative choice $h=100$ pc are shown as thin lines. We also show CMB anisotropy limits from FIRAS and sensitivities from PIXIE [21], direct detection limits from Xenon10 [22], Super-CDMS [23], and SENSEI [24], cosmic-ray limits from Ref. [10], and limits from DD of solar-reflected DM [7]. See the text for more details.

line between the detector and the neutrino beam, and by $75<K_{e} / \mathrm{MeV}<850$. DM accelerated by $\mathrm{CR}$ electrons induces a number of electron scatterings at MiniBooNE that we compute using Eq. (4) with the same energy and angular cuts, and a volume of 139 tons $\left(N_{e} \simeq 5 \times 10^{31}\right)$ that we infer from Ref. [28] as the one contained in a radius of $3.38 \mathrm{~m}$ (we conservatively interpret the $\nu-e$ cut "distance to wall" as referring to the distance from the spherical optical separation). We then integrate over a time of $124 \mathrm{sec}$, which we obtain by multiplying the observation time per pulse of $2 \mu \mathrm{sec}$ (third cut in Table III of Ref. [28]), with the total number of triggered pulses being $6.2 \times 10^{7}$. The latter is not explicitly given in Ref. [28], but we infer it as the total number of protons on target $\left(1.86 \times 10^{20}\right)$ divided by an average number of protons per pulse of $3 \times 10^{12}$ that again we infer from Ref. [28]. We include Earth attenuation using Eq. (6), where the amount of crust that DM goes across depends on $\theta_{e}$, the azimuthal angle $\phi_{e}$, and the depth of the booster $\simeq 6 \mathrm{~m}$ [29]. For simplicity we conservatively take the same value for the depth of MiniBooNE, corresponding to that of its center [30]. We finally multiply the signal events by 0.15 (the signal efficiency inferred from Ref. [28]) and impose the result to be smaller than the observed two events. The resulting constraint is displayed in Fig. 2. It extends to $\sigma_{e} \gtrsim 10^{-27} \mathrm{~cm}^{2}$, which we do not show, as that would require a treatment of DM scattering through the atmosphere, which goes beyond the purpose of this Letter. The 
analysis of more MiniBooNE data should allow us to close the small gap between the Super-K and MiniBooNE exclusions at $M_{\mathrm{DM}} \gtrsim 1 \mathrm{MeV}$. Our conservative MiniBooNE analysis, while admittedly rough, clearly demonstrates the point that cross sections larger than $10^{-29} \mathrm{~cm}^{2}$ are accessible at surface neutrino detectors.

Sensitivities at Super-K and DUNE.-We estimate them using the signal spatial information, i.e., the larger number of signal events expected from the direction of the Galactic Center. We integrate the signal over a cone with axis centered on the direction of the GC and opening angle of $10^{\circ}$, corresponding to the opening angle from Earth of the height of the cylinder assumed to contain the CR electrons, $h=1 \mathrm{kpc}$. In an actual search at neutrino experiments, the background could be estimated at Super-K using part of the space complementary to the cone as a control region, similar to what has been done in Ref. [17]. The uncertainty on the background would then be dominated by statistics, so that we estimate a $95 \%$ C.L. reach on light DM by imposing

$$
\left.\frac{N_{\text {DM }}}{\sqrt{N_{\text {DM }}+N_{\text {bkg }}}}\right|_{\text {a.h. }} ^{10^{\circ}}=2 .
$$

The subscript refers to the fact that we only use the fraction of the events above horizon, to be conservative with respect to the attenuation of the DM flux from Earth crossing.

In practice, we determine $N_{\mathrm{bkg}}^{\mathrm{SK}}$ at Super-K by multiplying the total events measured in the first energy bin [17] by the fraction of the sky over which we integrate $\simeq 0.01$, i.e., using the observed isotropy of the background. We determine $N_{\mathrm{bkg}}^{D}$ at DUNE assuming $200 \mathrm{kton}$ yr of data (to have the same number of electron years of Super-K), and using $d N_{\mathrm{bkg}}^{D} /\left.d t\right|_{10^{\circ}}=0.1$ event/kton yr [19]. We finally multiply the Super-K (DUNE) background events by 0.37 (0.32), i.e., by the time the $\mathrm{GC}$ is above the horizon, which we determine from Refs. [31,32]. For the signal we integrate Eq. (4) over the above cone (the signal fraction surviving is $\simeq 0.15$ ) and multiply by $0.37(0.32)$ at Super-K (DUNE). Other large volume detectors, like Hyper-K, also have promising sensitivities that can be determined as above.

The results are displayed in Fig. 2. The smallest values of the cross sections to which both Super-K and DUNE are sensitive are such that Earth would actually be transparent to DM. This would allow us, when performing an actual search, to gain sensitivity both from using events under the horison and by performing a full optimization of the region of integration (which we expect would have a wider opening angle in the direction of the galactic plane).

Other light-DM searches.-In Fig. 2 we also display constraints from standard direct detection [22-24], cosmic microwave background (CMB) [21], cosmic rays [10], and Sun reflection [7]. For simplicity we do not extend CR constraints below the $M_{\mathrm{DM}}$ range given in Ref. [10] because there the kinematical regime driving the shape of the line changes, and we stop Super-CDMS constraints at $\sigma_{e}=10^{-29} \mathrm{~cm}^{2}$ because of the $O(1) \mathrm{km}$ of rock above the detector.

The Sun constraints are given in Ref. [7] up to $\sigma_{e}=10^{-34} \mathrm{~cm}^{2}$, and down to $M_{\mathrm{DM}}=3 \mathrm{keV}$. We do not show them for $M_{\mathrm{DM}}<3 \mathrm{keV}$ because, in that range, the simple one-scattering regime with the core of the Sun is not enough to give the target electrons in the detectors enough energy to pass the cut of $0.19 \mathrm{keV}$ used in Ref. [7]. Therefore the study of those masses requires a treatment that goes beyond the purpose of this Letter. We also do not extend these limits above $\sigma_{e}=10^{-32} \mathrm{~cm}^{2}$ because they make the radial extension of the radiative area of the Sun become much larger than the related DM-electron interaction lengths, $R_{\text {rad }} \simeq 0.5 R_{\text {Sun }} \gg\left(\sigma_{e} n_{e}\right)^{-1}$, where, e.g., $n_{e} \approx 10^{23} \mathrm{~cm}^{-3}$ at the edge between the radiative and convective areas [33]. Therefore DM particles are expected to scatter several times in the radiative and convective regions, whose temperatures are much smaller than in the core of the Sun, leading to the expectation that the limits of Ref. [7] will be strongly affected.

We finally remark that, in the presence of additional interactions with the SM (e.g., with nucleons), the physics of DM escaping the Sun will become even more dependent on the outer Sun layers. Our limits from Super-K are instead more robust against assuming such an extra interaction (they would actually improve thanks to the extra up-scattered component from cosmic-ray protons) until it prevents DM from reaching the detector.

We do not show limits on $\sigma_{e}$ coming from the combination of CMB and big bang nucleosynthesis data [34-36], as they may be attenuated or evaded depending on other model assumptions, like the existence of additional dark radiation or annihilation channels for DM. Analogously, we do not show CMB constraints on annihilating DM, as they are more model dependent, and, for example, they are weak if DM annihilation is $p$-wave (see, e.g., Ref. [37]).

On concrete light-DM models. - A plethora of models of sub-GeV DM and dark sectors have recently been proposed: to name just a few, strongly interacting massive particles [38,39], elastically decoupling relics [40], light dark sectors and/or DM from supersymmetry [41], from leptogenesis [42], from the hierarchy problem [43,44], or demanded by observed anomalies, e.g., in $B$ decays $[45,46]$. Inspired by this rich model-building activity, we now briefly comment on the application of our results to some concrete models of light DM. A more detailed exploration of the following and other applications, while certainly interesting, goes beyond the purpose of this Letter.

An explicit example for which our strategy looks particularly promising is that of dark sectors with small mass splittings; see, e.g., Refs. [47-50]. These models can have sizable DM-electron interactions while evading limits from cosmology, SENSEI, Super-CDMS, etc., because in these energy domains the DM-electron 
scattering is inelastic. Our proposal avoids that limitation thanks to its larger energy regimes, and it therefore stands out as a prominent possibility to directly test such DM candidates.

We also studied for simplicity energy-independent contact interactions. The impact of these searches on other regimes can be grasped by observing that the energy exchanges that drive our sensitivities are of the order of the threshold of the neutrino detectors, $K_{e}>30-100 \mathrm{MeV}$. Therefore the performance of our proposal, with respect to other DD probes that rely on smaller energy exchanges (Sun reflection, CMB, Super-CDMS, etc.), would be better than what is displayed in Fig. 2 if $\sigma_{e}$ grows with increasing energy (e.g., as in the case of SM neutrinos), and it would be worse in the opposite case [e.g., for mediators much lighter than $O(100) \mathrm{MeV}$; see, e.g., Ref. [51] ].

Finally, if the relic particle $\chi$ interacting with electrons constitutes a subdominant component of DM, $f=$ $\Omega_{\chi} / \Omega_{\mathrm{DM}}<1$, then our constraints and sensitivities on $\sigma_{e}$ are relaxed by $\sqrt{f}$, unlike the more severe rescaling by $f$ of other DD probes.

Conclusions and outlook.-The results presented in this Letter demonstrate that large volume neutrino experiments have a promising potential to probe unexplored regimes of light-DM interactions with the SM. This physics case relies on our novel proposal to test the energetic DM component that is unavoidably generated by scatterings with $\mathrm{CR}$ electrons in the Galaxy. The conservative limit we set using public Super-K data excludes previously allowed wide regions of parameter space, and that could be improved if a dedicated search would be performed in existing data at Super-K; see Fig. 2. The prospects of other large neutrino experiments, like Hyper-K and DUNE, also look bright.

Thinking about possible future directions, going to lower electron energy thresholds would increase the signal by allowing experiments to be sensitive to a larger fraction of the up-scattered DM (see Fig. 1). That would pose the challenge of dealing with much larger backgrounds, e.g., from solar neutrinos [52]. While we do not explore this regime further here, we encourage the experimental collaborations to pursue that direction, for example, by employing the peculiar modulation of the signal (from the daily rotation of the $\mathrm{GC}$ direction).

We thank Kfir Blum and Luc Darmé for the useful discussions and Christopher Cappiello for pointing out a numerical glitch in our previous version of Figs. 1 and 2, which does not affect our conclusions. Y. E. is supported in part by JSPS KAKENHI Grant No. JP18J00540. F. S. is supported in part by PIER Seed Project funding (Project ID No. PIF-2017-72).

Note added.-Recently, Ref. [53] appeared, proposing the same idea that DM up-scattered by cosmic rays can give observable effects in Earth detectors. That work is complementary to ours in that it focuses on DM-nucleon interactions and on signals at detectors like Xenon-1T, while we focus on DM-electron interactions and on signals at large neutrino experiments.

[1] M. W. Goodman and E. Witten, Phys. Rev. D 31, 3059 (1985).

[2] D. S. Akerib et al. (LUX Collaboration), Phys. Rev. Lett. 118, 021303 (2017).

[3] X. Cui et al. (PandaX-II Collaboration), Phys. Rev. Lett. 119, 181302 (2017).

[4] E. Aprile et al. (XENON Collaboration), Phys. Rev. Lett. 121, 111302 (2018).

[5] G. F. Giudice, in From My Vast Repertoire ...: Guido Altarelli's Legacy, edited by A. Levy, S. Forte, and G. Ridolfi (World Scientific, Singapore, 2019), pp. 267-292.

[6] M. Battaglieri et al., in Proceedings of U.S. Cosmic Visions: New Ideas in Dark Matter College Park, MD, 2017, arXiv: 1707.04591.

[7] H. An, M. Pospelov, J. Pradler, and A. Ritz, Phys. Rev. Lett. 120, 141801 (2018).

[8] T. Emken, C. Kouvaris, and N. G. Nielsen, Phys. Rev. D 97, 063007 (2018).

[9] K. Agashe, Y. Cui, L. Necib, and J. Thaler, J. Cosmol. Astropart. Phys. 10 (2014) 062.

[10] C. V. Cappiello, K. C. Y. Ng, and J. F. Beacom, Phys. Rev. D 99, 063004 (2019).

[11] M. J. Boschini et al., Astrophys. J. 854, 94 (2018).

[12] J. F. Navarro, C. S. Frenk, and S. D. M. White, Astrophys. J. 462, 563 (1996).

[13] See Supplemental Material at http://link.aps.org/ supplemental/10.1103/PhysRevLett.122.181802, which includes Refs. [14-16], for DM kinetic energy distribution.

[14] J. D. Lewin and P. F. Smith, Astropart. Phys. 6, 87 (1996).

[15] M. Kuhlen, N. Weiner, J. Diemand, P. Madau, B. Moore, D. Potter, J. Stadel, and M. Zemp, J. Cosmol. Astropart. Phys. 02 (2010) 030.

[16] M. Lisanti, in Proceedings of the Theoretical Advanced Study Institute in Elementary Particle Physics: New Frontiers in Fields and Strings (TASI 2015): Boulder, CO, 2015, edited by J. Polchinski, P. Vieira, and O. DeWolfe (World Scientific, Singapore, 2017), pp. 399-446.

[17] C. Kachulis et al. (Super-Kamiokande Collaboration), Phys. Rev. Lett. 120, 221301 (2018).

[18] R. Acciarri et al. (DUNE Collaboration), arXiv:1512.06148.

[19] L. Necib, J. Moon, T. Wongjirad, and J. M. Conrad, Phys. Rev. D 95, 075018 (2017).

[20] T. Emken and C. Kouvaris, Phys. Rev. D 97, 115047 (2018).

[21] Y. Ali-Haïmoud, J. Chluba, and M. Kamionkowski, Phys. Rev. Lett. 115, 071304 (2015).

[22] R. Essig, A. Manalaysay, J. Mardon, P. Sorensen, and T. Volansky, Phys. Rev. Lett. 109, 021301 (2012).

[23] R. Agnese et al. (SuperCDMS Collaboration), Phys. Rev. Lett. 121, 051301 (2018).

[24] M. Crisler, R. Essig, J. Estrada, G. Fernandez, J. Tiffenberg, M. S. Haro, T. Volansky, and T.-T. Yu (SENSEI Collaboration), Phys. Rev. Lett. 121, 061803 (2018). 
[25] O. Adriani et al. (CALET Collaboration), Phys. Rev. Lett. 119, 181101 (2017).

[26] D. Kim, K. Kong, J.-C. Park, and S. Shin, J. High Energy Phys. 08 (2018) 155.

[27] A. Chatterjee, A. De Roeck, D. Kim, Z. G. Moghaddam, J.-C. Park, S. Shin, L. H. Whitehead, and J. Yu, Phys. Rev. D 98, 075027 (2018).

[28] A. A. Aguilar-Arevalo et al. (MiniBooNE DM Collaboration), Phys. Rev. D 98, 112004 (2018).

[29] FNAL, https://www.fnal.gov/pub/visiting/map/booster.html (2018).

[30] A. A. Aguilar-Arevalo et al. (MiniBooNE Collaboration), Nucl. Instrum. Methods Phys. Res., Sect. A 599, 28 (2009).

[31] T. P. Robitaille, E. J. Tollerud, P. Greenfield, M. Droettboom, E. Bray, T. Aldcroft, M. Davis, A. Ginsburg, A. M. PriceWhelan et al. (Astropy Collaboration), Astron. Astrophys. 558, A33 (2013).

[32] A. M. Price-Whelan, B. M. Sipőcz, H. M. Günther, P. L. Lim, S. M. Crawford, S. Conseil, D. L. Shupe, M. W. Craig, N. Dencheva et al. (Astropy Collaboration), Astron. J. 156, 18 (2018).

[33] NASA, https://solarscience.msfc.nasa.gov/interior.shtml (2015).

[34] C. Boehm, M. J. Dolan, and C. McCabe, J. Cosmol. Astropart. Phys. 08 (2013) 041.

[35] K. M. Nollett and G. Steigman, Phys. Rev. D 89, 083508 (2014).

[36] K. M. Nollett and G. Steigman, Phys. Rev. D 91, 083505 (2015).

[37] H. Liu, T. R. Slatyer, and J. Zavala, Phys. Rev. D 94, 063507 (2016).
[38] Y. Hochberg, E. Kuflik, T. Volansky, and J. G. Wacker, Phys. Rev. Lett. 113, 171301 (2014).

[39] S.-M. Choi, Y. Hochberg, E. Kuflik, H. M. Lee, Y. Mambrini, H. Murayama, and M. Pierre, J. High Energy Phys. 10 (2017) 162.

[40] E. Kuflik, M. Perelstein, N. Rey-Le Lorier, and Y.-D. Tsai, Phys. Rev. Lett. 116, 221302 (2016).

[41] N. Arkani-Hamed and N. Weiner, J. High Energy Phys. 12 (2008) 104.

[42] A. Falkowski, E. Kuflik, N. Levi, and T. Volansky, Phys. Rev. D 99, 015022 (2019).

[43] N. Fonseca and E. Morgante, arXiv:1809.04534.

[44] A. Banerjee, H. Kim, and G. Perez, arXiv:1810.01889.

[45] F. Sala and D. M. Straub, Phys. Lett. B 774, 205 (2017).

[46] F. Sala, Nucl. Part. Phys. 303-305, 14 (2018).

[47] D. Kim, J.-C. Park, and S. Shin, Phys. Rev. Lett. 119, 161801 (2017).

[48] L. Darmé, S. Rao, and L. Roszkowski, J. High Energy Phys. 03 (2018) 084.

[49] G. F. Giudice, D. Kim, J.-C. Park, and S. Shin, Phys. Lett. B 780, 543 (2018).

[50] L. Darmé, S. Rao, and L. Roszkowski, J. High Energy Phys. 12 (2018) 014.

[51] R. Barkana, N. J. Outmezguine, D. Redigolo, and T. Volansky, Phys. Rev. D 98, 103005 (2018).

[52] A. Gutlein, C. Ciemniak, F. von Feilitzsch, N. Haag, M. Hofmann, C. Isaila, T. Lachenmaier, J.-C. Lanfranchi, L. Oberauer, and S. Pfister, Astropart. Phys. 34, 90 (2010).

[53] T. Bringmann and M. Pospelov, arXiv:1810.10543 [Phys. Rev. Lett. (to be published)]. 